\title{
Pancreatic fatty cleft mimicking a pancreatic lipoma: A diagnostic pitfall
}

\author{
Mehmet Haydar Atalar ${ }^{1}$
}

${ }^{1}$ Cumhuriyet University School of Medicine, Department of Radiology, Sivas, Turkey

Corresponding author: Mehmet Haydar Atalar, Cumhuriyet University School of Medicine, Department of Radiology, Sivas, Turkey

E-mail: mhatalar@gmail.com

Received/Accepted: September 20, 2017 / December 28, 2017

Conflict of interest: There is not a conflict of interest.

To the editor,

The pancreas is included and well demonstrated on every cross-sectional imaging of the abdomen. A normal pancreas has a lobulated surface. Lobular contour abnormalities of the pancreas can be seen at rates of up to $35 \%$ in healthy individuals. Pancreatic clefts are anatomic variants that are likely to cause most diagnostic confusion when detected incidentally in patients being imaged for unrelated reasons (may be mistaken for a mass) or after trauma (may be mistaken for a fracture). Occasionally, peripancreatic fat "trapped" within a deep cleft or fold between these lobulations may mimic a mass (particularly lipoma) or laceration/transection at cross-sectional imaging. The majority of these anatomical variants have no clinical significance, although they need to be recognized as anatomical variants by the radiologist ${ }^{1-3}$.

A 57-year-old man was referred from an outside center where he was found to have a lipoma at the tail of pancreas detected by a CT examination that was performed to assess a mass lesion at the location of the right adrenal gland. A contrast enhanced thin section abdominal CT performed to evaluate the pancreas revealed a nodular area at fat density (HU: -68) showing continuity with the peripancreatic fat tissue inside a deep pancreatic cleft at the tail section of the pancreas. Based on these findings, a pancreatic lipoma was excluded. The other sections of the pancreas and both surrenal glands were of normal appearance. The laboratory findings of the patient (including serum pancreatic enzymes) were within normal limits.

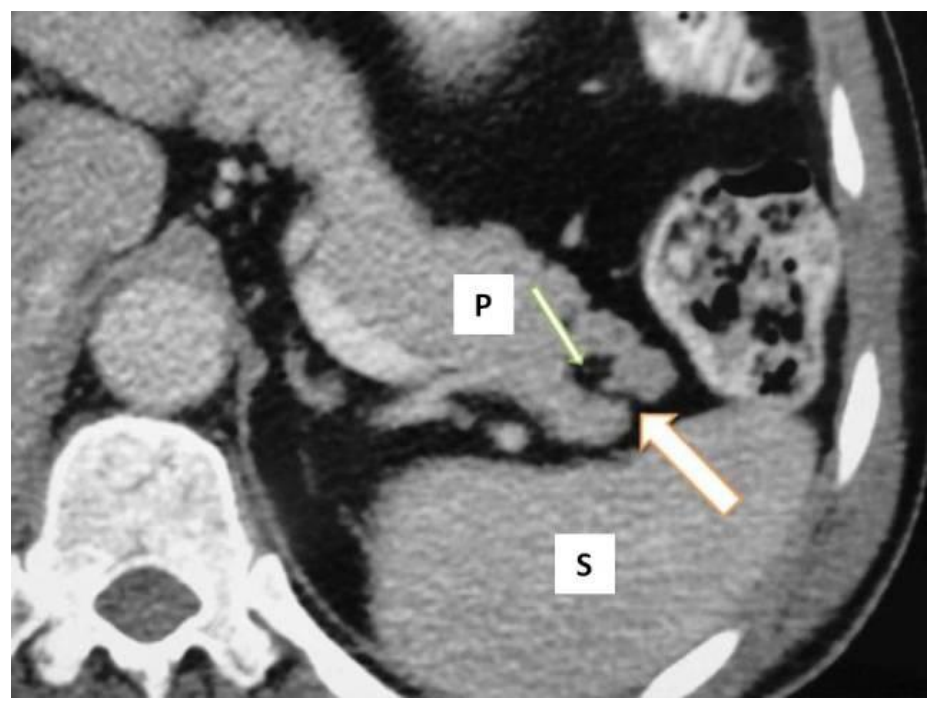

Figure 1. Axial contrast enhanced thin section CT image shows the apparent mass (thin arrow) is of fat density and is contiguous anteriorly with the fat around the pancreas, consistent with fat within a deep pancreatic cleft (thick arrow). (Abbrevations: P; Pancreas, S; Spleen) 
Pancreatic clefts are usually detected at the junction of the pancreatic neck and body. Peripancreatic fat may be invaginate into pancreatic tissue through these clefts. Histologically, pancreatic fatty clefts represent peripancreatic fat entrapped inside a normal pancreatic parenchyma, and they are observed at sites where vascular structures penetrating pancreatic parenchyma reside in. Their incidence increases with age. They may be mistaken for a pancreatic fracture in a traumatized patient. The diagnosis of a pancreatic fatty cleft should be considered for any apparent small pancreatic lesion that abuts the gland surface, and can usually be confirmed by examining thin section CT images. MRI sequences with and without fat saturation may also be helpful for diagnosis ${ }^{1-4}$.

\section{REFERENCES}

1. Borghei P, Sokhandon F, Shirkhoda A, Morgan DE. Anomalies, anatomic variants, and sources of diagnostic pitfalls in pancreatic imaging. Radiology. 2013; 266: 28-36.

2. Heuck A, Maubach PA, Reiser M, et al. Agerelated morphology of the normal pancreas on computed tomography. Gastrointest Radiol. 1987; 12: 18-22.

3. Alexander LF. Congenital pancreatic anomalies, variants, and conditions. Radiol Clin North Am. 2012; 50: 487-98.

4. Nijs E, Callahan MJ, Taylor GA. Disorders of the pediatric pancreas: imaging features. Pediatr Radiol. 2005; 35: 358-73. 